\title{
Prevalência de transtornos mentais e comportamentais nas instituições públicas federais de Rondônia
}

\author{
Vanderléia de Lurdes Dal Castel Schlindwein ${ }^{1}$, Paulo Rogério Morais ${ }^{2}$ \\ Universidade Federal de Rondônia (Porto Velho, RO)
}

\begin{abstract}
Este artigo tem por objetivo avaliar a prevalência de transtornos mentais e comportamentais nas instituições públicas federais, registrados no Subsistema Integrado de Atenção à Saúde do Servidor Público Federal/SIASS de Rondônia. O estudo do tipo descritivo-exploratório transversal propôs-se analisar os prontuários das licenças no trabalho por motivos de saúde, enquadradas no grupo F, de acordo com a Classificação Internacional de Doenças CID-10. Foram estudadas vinte e três instituições públicas federais deste estado no ano de 2011. Foram concedidos 3.079 afastamentos por licença saúde, aos servidores públicos federais sendo que destes, 296 (9,6\%) foram motivados por transtornos mentais e comportamentais. Observou-se diferenças na prevalência de afastamentos entre os sexos, as licenças saúde concedidas às mulheres $(70,6 \%)$ foram superiores ao número concedido aos homens (29\%), entretanto houve diferença nos diagnósticos entre os sexos. $\mathrm{O}$ uso de substâncias psicoativas foi superior entre os homens $(10,3 \%)$ em relação às mulheres $(1,4 \%)$, já para as mulheres prevaleceram os transtornos de humor associados aos transtornos neuróticos $(11,4 \%)$ em relação aos homens $(1,5 \%)$.
\end{abstract}

Palavras-chave: Saúde do Trabalhador, Transtornos mentais, Setor público e Licença médica.

Prevalence of mental and behavioral disorders in federal public institutions in the state of Rondônia, Brazil

This article aims to assess the prevalence of mental and behavioral disorders in federal public institutions, registered in the Integrated Care Subsystem for the SIASS Federal Public Service Health of the state of Rondônia. This descriptive and exploratory study aimed to examine the medical records of licenses at work due to health reasons, framed in group F, according to the International Classification of Diseases ICD-10. Three thousand and seventy-nine absences were granted for sick leave, and of these, 296 (9,6\%) were motivated by mental and behavioral disorders. Differences in the prevalence of clearances between the sexes were observed: sick leaves granted to women $(70,6 \%)$ were higher than the number granted to men (29\%), however there was no difference in diagnoses between the sexes. In short, the use of psychoactive substances was higher among men $(10,3 \%)$ than women $(1,4 \%)$, while for women prevailed mood disorders associated with neurotic issues $(11,4 \%)$ when compared to men $(1,5 \%)$.

Keywords: Workers Health, Mental Disorders, Public Sector e Sickness Leave.

\section{Introdução}

A prevalência dos afastamentos por Transtornos Mentais e Comportamentais relacionados ao trabalho representa um problema de saúde pública. De acordo com dados do Instituto Nacional de Seguridade Social/INSS, no Brasil os transtornos mentais estão na terceira colocação das causas de concessão de benefícios previdenciários (Brasil, 2001).

O aumento do estresse relacionado ao trabalho vem sendo destacado como uma das importantes causas de transtornos mentais e comportamentais, dessa forma, propiciando o aumento do absenteísmo no trabalho (McDaid, 2008) e acarretando altos custos às economias em todo o mundo (Seligmann-Silva, 2009).

De acordo com a Organização Internacional do Trabalho/OIT (2010), a cada ano 2\% da população mundial sofre com algum tipo de enfermidade, seja ela física ou mental. Cerca de 160 milhões de pessoas por ano se tornam vítimas de doenças no local de trabalho. Partindo

1 Docente do Departamento de Psicologia (DEPSI) e do Programa de Pós-Graduação em Psicologia (MAPSI) da Universidade Federal de Rondônia, coordenadora do Centro de Estudo e Pesquisas em Saúde Mental e Trabalho na Amazônia/CEPEST/ Rondônia.

2 Docente do Departamento de Psicologia (DEPSI), coordenador de pesquisa e extensão do Centro Regional de Referência em Álcool e outras Drogas (CRR/SENAD/UNIR). 
desses dados, a Organização aponta que a cada 15 segundos, um trabalhador morre decorrente de doenças relacionadas à atividade laboral, e destaca, no relatório de 2010, os riscos emergentes e a necessidade de construir novos modelos de prevenção no mundo do trabalho em transformação. Este relatório destaca que os riscos decorrentes das novas tecnologias e novos processos de trabalho estão associados aos fatores psicossociais e de estresse no trabalho.

Os vínculos entre o trabalho e adoecimento mental vêm ganhando visibilidade crescente em vários estudos, entre os quais corroboram essa relação ao apresentar a justificativa da relação causal entre agravos à saúde mental e o trabalho (Vieira, 2009; Seligmann-Silva, 2010; Borges, 2010; Vieira \& Jardim, 2010, Glina, 2010 e outros). Além disso, o reconhecimento formal dos transtornos mentais relacionados ao trabalho está no Manual de Doenças Relacionadas ao Trabalho (Ministério da Saúde, 2001). A relação entre adoecimento mental e trabalho é compreendido por Seligmann-Silva (2011, p. 35), "o trabalho, conforme a situação, tanto poderá fortalecer a saúde mental quanto vulnerabilizá-la e mesmo gerar distúrbios que se expressarão coletivamente e no plano individual".

Acredita-se que este estudo poderá subsidiar o desenvolvimento de ações voltadas à saúde mental no trabalho, especialmente no Estado em que foi realizado o estudo, possibilitando um eficaz planejamento das ações em políticas públicas da saúde do servidor público federal, direcionadas acerca dos fatores psicossociais que contribuem para a saúde ou levam ao afastamento no trabalho nas instituições públicas federais da região amazônica.

Nessa perspectiva, o estudo objetivou avaliar a prevalência de afastamentos por transtornos mentais e comportamentais enquadrados no grupo F do CID-10 de servidores públicos federais do Estado de Rondônia, registrados no Subsistema Integrado de Atenção à Saúde do Servidor Público Federal/SIASS, no ano de 2011.

\section{Metodologia}

Trata-se de um estudo do tipo descritivo-exploratório transversal no qual se buscou, através de fontes documentais, dados quantitativos dos afastamentos dos servidores públicos federais de vinte e três instituições públicas do Estado por licença médica no trabalho registrados no Subsistema Integrado de Atenção à Saúde do Servidor Público Federal/SIASS/RO33, no período de janeiro a dezembro de 2011.

Os dados foram obtidos a partir dos arquivos disponíveis nos protocolos da perícia médica do setor responsável pelo registro. A partir disso realizou-se uma investigação e sistematização das informações contidas nos protocolos das indicações médicas de afastamentos conforme descrição da Classificação Estatística Internacional de Doenças e Problemas Relacionados à Saúde Décima Revisão (CID-10), descritos no capítulo V - Transtornos Mentais e Comportamentais (F00-F99). Analisaram-se as licenças concedidas pela perícia médica do SIASS de vinte e três (23) órgãos públicos federais que faziam parte do termo de cooperação técnica. Vale ressaltar que duas instituições não tinham registro de afastamentos por transtornos mentais no ano de 2011. As variáveis analisadas foram: sexo, a causa de afastamento segundo os tipos de transtornos mentais e comportamentais representados pelo código F da CID-10, instituição, idade, diagnóstico/CID e tempo concedido de licença por doença e as prorrogações da licença.

3 O acordo de cooperação técnica do primeiro núcleo do SIASS/Superintendência Estadual da FUNASA/SUEST-RO, foi celebrado no final de 2010 entre 25 órgãos federais no estado de Rondônia, com sede em Porto Velho. Sua ação principal é dar assistência à saúde, desde ações de prevenção, detecção precoce e o tratamento de doenças e, ainda, a reabilitação da saúde do servidor, como realizar a perícia oficial integrando ações médicas e odontológicas com o objetivo de avaliar o estado de saúde do servidor para o exercício de suas atividades laborais, e ações de promoção, prevenção e acompanhamento da saúde (Decreto n 6.833 , de 29 de Abril de 2009). 
Os transtornos mentais e comportamentais registrados nos prontuários do SIASS foram divididos em oito (8) grupos - entre F00 e F89 -, de acordo com a CID-10. São eles: Transtornos mentais orgânicos, inclusive os sintomáticos (F00-F09); Transtornos mentais e comportamentais devido ao uso de substância psicoativa (F10-F19); Esquizofrenia, transtornos esquizotípicos e transtornos delirantes (F20-F29); Transtornos do humor [afetivos] (F30-F39); Transtornos neuróticos, transtornos relacionados com o estresse e transtornos somatoformes (F40-F48); Síndromes comportamentais associadas a disfunções fisiológicas e a fatores físicos (F50-F59); Distorções da personalidade e do comportamento adulto (F60-F69); Retardo mental (F70-F79); Transtornos do desenvolvimento psicológico (F80-F89).

Para fins de análise, foi realizada uma categorização das instituições públicas federais do estado em grupos. Para isto, foram agrupadas em seis áreas, distribuídas por similaridade e finalidade. Sendo assim, tais áreas foram reagrupadas em seis: (1) o grupo da educação abrangeu duas instituições; (2) a área da segurança pública ficou com quatro instituições; (3) o grupo da saúde e proteção social integrou três instituições públicas; (4) a engenharia, agricultura e pecuária representaram cinco instâncias públicas; (5) recursos florestais e engenharia florestal somaram quatro instituições e as (6) jurídico administrativas foram cinco. Esse agrupamento facilitou a compreensão não apenas de algumas variáveis de cada instituição como também permitiu a comparação de indicadores, como sexo, motivo da licença/CID e dias de afastamento e comorbidades relacionadas aos afastamentos.

As análises estatísticas foram realizadas com o programa Statistical Package for the Social Sciences, versão 20.0 (SPSS-20.0). Para a comparação de dados nominais utilizou-se o teste do Qui-quadrado e para as análises das variáveis com mensuração intervalar foi utilizada o teste de Kruskal-Wallis, seguido do teste U de Mann-Whitney quando necessário. Em todos os casos, o nível de significância foi de 5\% (Morais, 2007).

O estudo foi autorizado pelo coordenador do SIASS/RO e aprovado pelo Comitê de Ética em Pesquisa da Universidade Federal de Rondônia sob o protocolo no 439280/2011-13, em atendimento à Resolução CNS no 196/96, do Conselho Nacional de Saúde.

\section{Resultados}

Em Rondônia, no ano de 2011 foram concedidas 3.079 licenças saúde a servidores públicos federais. Desses afastamentos, 296 (9,6\%) foram motivadas por transtornos mentais e comportamentais, representados na CID-10 pelas doenças do código F. A amostra foi composta por $194(70,6)$ mulheres e 87 sujeitos masculinos $(29,4 \%)$ do total de transtornos mentais. Vale ressaltar que em 17 (5,7\%) prontuários não especificavam o sexo do sujeito. A média de idade geral dos servidores afastados é de 47,9 99,4 anos (média \pm DP).

Os dados da Tabela 1 foram analisados com o teste do qui-quadrado, desconsiderando os prontuários nos quais não constava a idade dos indivíduos (43,7\%), demonstrando que o número de trabalhadores com idades entre 50 e 59 anos que foram afastados do trabalho por TM é estatisticamente superior às demais faixas etárias $\left(c^{2}=243,2 ; \mathrm{df}=4 ; \mathrm{p}<0,001\right)$.

Tabela 1. Distribuição das faixas etárias dos trabalhadores afastados por Transtornos mentais e comportamentais.

\begin{tabular}{lll}
\hline Faixa etária & Frequência & $\%$ \\
\hline $19-29$ & 11 & 3,7 \\
$30-39$ & 25 & 8,5 \\
$40-49$ & 14 & 4,7 \\
\hline
\end{tabular}




\begin{tabular}{lll}
\hline \hline Faixa etária & Frequência & $\%$ \\
\hline $50-59$ & 111 & 37,6 \\
$60-69$ & 5 & 1,7 \\
Dado indisponível & 130 & 43,7 \\
Total & 296 & 100,0 \\
\hline \hline
\end{tabular}

Fonte: Elaborada pelos autores, a partir dos dados obtidos no Subsistema Integrado de Atenção à Saúde do Servidor Público Federal/ SIASS/RO.

Na Tabela 2, observa-se que a comparação dos totais de afastamentos dos grupos de instituições por meio do teste do qui-quadrado demonstrou haver diferença estatisticamente significativa entre os grupos $\left(c^{2}=713,7 ; d f=5 ; p<0,001\right)$. O grupo formado pelas instituições das áreas de "Saúde e Proteção Social" apresentou um número maior de afastamentos por licença saúde do que os demais grupos $\left(c^{2}=35,8 ; d f=1 ; p<0,001\right)$. Os grupos "Engenharia, Agricultura e Pecuária" e "Recursos Florestais e Engenharia Ambiental" foram os que apresentaram menor número e afastamentos motivados por questões de saúde $\left(c^{2}=199,3\right.$; $\mathrm{df}=1 ; \mathrm{p}<0,001)$ e os grupos das áreas "Jurídico Administrativas", "Segurança Pública" e "Educação" não diferiram entre si quanto ao número total de afastamentos por licença saúde $\left(c^{2}=1,7 ; \mathrm{df}=2 ; \mathrm{p}>0,05\right)$.

Também foram detectadas diferenças estatisticamente significativas nos afastamentos motivados por diagnósticos de transtornos mentais $\left(\mathrm{c}^{2}=68,4 ; \mathrm{df}=5 ; \mathrm{p}<0,001\right)$. $\mathrm{O}$ número de licença saúde por causa de transtornos mentais foi estatisticamente maior no grupo das instituições das áreas de "Saúde e Proteção Social" ( $\left.c^{2}=14,8 ; d f=1 ; p<0,001\right)$. O número de afastamentos por transtornos mentais foi estatisticamente menor no grupo da "Engenharia, Agricultura e Pecuária" e "Recursos Florestais e Engenharia Ambiental" ( $\left.c^{2}=4,0 ; \mathrm{df}=1 ; \mathrm{p}<0,05\right)$.

Tabela 2. Número de licenças médicas por motivos de saúde classificadas na CID-10 do grupo F dos Transtornos mentais e comportamentais de vinte e três instituições públicas federais de Rondônia, agrupados por áreas, no ano de 2011.

\begin{tabular}{lll}
\hline $\begin{array}{l}\text { Grupos de instituições } \\
\text { por áreas }\end{array}$ & Número de licença saúde & $\begin{array}{l}\text { Número de licenças por } \\
\text { Transtornos mentais e } \\
\text { Comportamentais }\end{array}$ \\
\hline Saúde e Proteção Social & 867 & 168 \\
Recursos Florestais e Engenharia & 183 & 17 \\
Ambiental & 589 & 33 \\
Jurídico Administrativas & 611 & 35 \\
Segurança Pública & 194 & 8 \\
Engenharia, Agricultura e Pecuária & 635 & 18 \\
Educação & 3.079 & 279 \\
\hline Total & & 5940 \\
\hline
\end{tabular}

Fonte: Elaborada pelos autores, a partir dos dados obtidos no Subsistema Integrado de Atenção à Saúde do Servidor Público Federal/ SIASS/RO.

Na Figura 1, a análise do qui-quadrado demonstrou haver diferença estatisticamente significativa entre os grupos na frequência de afastamentos decorrentes de transtornos mentais $\left(c^{2}=57,7 ; \mathrm{df}=5 ; \mathrm{p}<0,001\right)$. A proporção de indivíduos que precisaram se afastar de suas atividades laborais em virtude de transtornos mentais foi estatisticamente maior no grupo "Saúde e Proteção Social" ( $\left.c^{2}=12,1 ; \mathrm{df}=1 ; \mathrm{p}<0,001\right)$. Os demais grupos não apresentaram diferenças significativas 
entre si, exceto os grupos "Engenharia, Agricultura e Pecuária” e "Recursos Florestais e Engenharia Ambiental" que apresentaram uma proporção estatisticamente inferior aos grupos "Segurança Pública" e "Saúde e Proteção Social" ( $\left.c^{2}=4,4 ; \mathrm{df}=1 ; \mathrm{p}<0,05\right)$.

Quando os dados são estratificados com base no sexo, observa-se que a proporção de indivíduos do sexo feminino que tiveram afastamento motivado por transtornos mentais difere entre os grupos $\left(c^{2}=59,1 ; d f=5 ; p<0,001\right)$. No grupo, "Saúde e Proteção Social" a proporção de mulheres afastadas por transtornos mentais foi estatisticamente superior ao observado nos demais grupos $\left(c^{2}=7,6 ; d f=2 ; p<0,05\right)$. Não foi detectada diferença significativa entre os grupos "Educação" e "Jurídico Administrativas" ( $\left.c^{2}=1,0 ; d f=1 ; p>0,05\right)$. Os grupos que apresentaram menor número de mulheres afastadas por transtornos mentais foram "Engenharia, Agricultura e Pecuária", "Recursos Florestais e Engenharia Ambiental" e "Segurança Pública" ( $c^{2}=3,9 ; \mathrm{df}=1$; $\mathrm{p}<0,05)$.

O teste do qui-quadrado também demonstrou haver diferença significativa na proporção de homens afastados por transtornos mentais entre os grupos $\left(c^{2}=26,1 ; d f=1 ; p<0,001\right)$. A proporção de homens afastados por transtornos mentais dos grupos "Segurança Pública", "Saúde e Proteção Social" e "Educação" foi estatisticamente superior à dos demais grupos $\left(c^{2}=6,4 ; \mathrm{df}=1\right.$; p <0,05), e os grupos da "Engenharia, Agricultura e Pecuária", "Recursos Florestais e Engenharia Ambiental" e "Jurídico Administrativas" não diferiram entre si ( $\left.c^{2}=0,5 ; \mathrm{df}=1 ; \mathrm{p}>0,05\right)$.

A comparação dos afastamentos masculinos e femininos nos grupos demonstrou que nos grupos "Engenharia, Agricultura e Pecuária" e "Recursos Florestais e Engenharia Ambiental" não há diferença entre as proporções de homens e mulheres que se afastaram por transtornos mentais. No entanto, a proporção de homens afastados por transtornos mentais foi estatisticamente maior do que a de mulheres nos grupos "Educação" $\left(c^{2}=4,6 ; d f=1 ; p<0,05\right)$ e "Segurança Pública" $\left(\mathrm{c}^{2}=8,8 ; \mathrm{df}=1 ; \mathrm{p}<0,005\right)$. A proporção de mulheres afastadas por transtornos mentais foi maior que a dos homens nos grupos "Saúde e Proteção Social" ( $c^{2}=8,8$; $\mathrm{df}=1 ; \mathrm{p}<0,05)$ e "Jurídico Administrativa" $\left(\mathrm{c}^{2}=7,0 ; \mathrm{df}=1 ; \mathrm{p}<0,05\right)$ estatisticamente superior à dos demais grupos, e os grupos de "Engenharia, Agricultura e Pecuária", "Recursos Florestais e Engenharia Ambiental”.

Figura 1. Frequência dos afastamentos por Transtorno mental e comportamental por sexo nas vinte e três instituições públicas federais de Rondônia, no período de janeiro a dezembro de 2011.

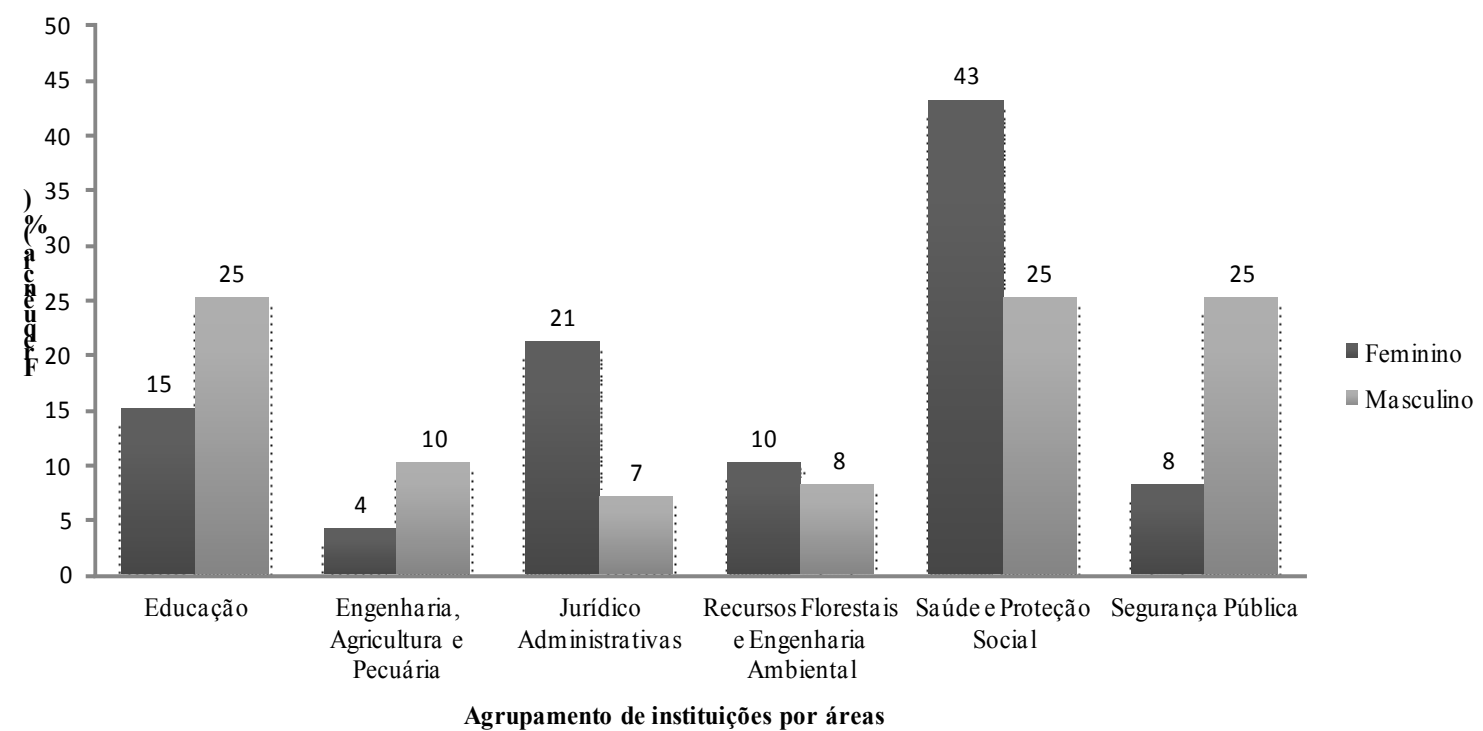

Fonte: Elaborada pelos autores, a partir dos dados obtidos no Subsistema Integrado de Atenção à Saúde do Servidor Público Federal/ SIASS/RO. 
Como se observa na Tabela 3, os transtornos mentais mais prevalentes em ambos os grupos foram os Transtornos de Humor (F30 - F39) e Transtornos neuróticos, transtornos relacionados com o estresse e transtornos somatoformes (F40 - F48). O teste do qui-quadrado demonstrou que o número de afastamentos motivados por transtornos de humor foi estatisticamente superior ao de afastamentos por transtornos neuróticos nos dois grupos $\left(c^{2}=8,6 ; \mathrm{df}=1 ; \mathrm{p}<0,005\right)$. Na comparação entre os sexos foram observadas diferenças estatisticamente significativas para $\mathrm{o}$ diagnóstico de Transtornos mentais e comportamentais devido ao uso de substância psicoativa e para os quadros mistos de depressão e ansiedade, sendo que no caso dos transtornos decorrentes do uso de substâncias psicoativas a proporção de homens é superior à de mulheres $\left(\mathrm{c}^{2}=7,4 ; \mathrm{df}=1\right.$; $\mathrm{p}<0,05)$ e, no caso de transtornos de humor associados a transtornos neuróticos, as mulheres apresentam mais estes diagnósticos do que os homens $\left(\mathrm{c}^{2}=6,2 ; \mathrm{df}=1 ; \mathrm{p}<0,05\right)$.

Tabela 3. Prevalência de afastamentos de saúde por Transtornos mentais e comportamentais de servidores públicos, segundo capítulos da CID-10, de vinte e três instituições públicas federais de Rondônia, no período de janeiro a dezembro de 2011.

\begin{tabular}{|c|c|c|}
\hline $\begin{array}{l}\text { Grupo Diagnóstico do CID } \\
\text { por áreas }\end{array}$ & $\begin{array}{l}\text { Percentual (\%) de } \\
\text { afastamentos entre mulheres }\end{array}$ & $\begin{array}{l}\text { Percentual (\%) de } \\
\text { afastamentos entre homens }\end{array}$ \\
\hline $\begin{array}{l}\text { Transtornos mentais orgânicos, inclusive os sintomáticos } \\
\text { (F00-F09) }\end{array}$ & 2,1 & 0 \\
\hline $\begin{array}{l}\text { Transtornos mentais e comportamentais devido ao uso de } \\
\text { substância psicoativa (F10-F19) }\end{array}$ & 1,4 & $10,3 *$ \\
\hline $\begin{array}{l}\text { Esquizofrenia, transtornos esquizotípicos e transtornos delirantes } \\
\text { (F20-F29) }\end{array}$ & 0,7 & 2,9 \\
\hline Transtornos do humor [afetivos] (F30-F39) & 52,1 & 44,1 \\
\hline $\begin{array}{l}\text { Transtornos neuróticos, transtornos relacionados com o estresse } \\
\text { e transtornos somatoformes (F40-F48) }\end{array}$ & 29,5 & 36,7 \\
\hline $\begin{array}{l}\text { Síndromes comportamentais associadas a disfunções fisiológicas e } \\
\text { a fatores físicos (F50-F59) }\end{array}$ & 0,7 & 3,0 \\
\hline $\begin{array}{l}\text { Distorções da personalidade e do comportamento adulto } \\
\text { (F60-F69) }\end{array}$ & 1,4 & 1,5 \\
\hline Retardo mental (F70-F79) & 0 & 0 \\
\hline Transtornos do desenvolvimento psicológico (F80-F89) & 0,7 & 0 \\
\hline $\begin{array}{l}\text { Transtornos do humor [afetivos] + Transtornos neuróticos, } \\
\text { transtornos relacionados com o estresse e transtornos } \\
\text { somatoformes }\end{array}$ & $11,4^{*}$ & 1,5 \\
\hline Total & 100 & 100 \\
\hline
\end{tabular}

* Diferença estatisticamente significativa entre os sexos $(\mathrm{p}<0,05)$

Fonte: Elaborada pelos autores, a partir dos dados obtidos no Subsistema Integrado de Atenção à Saúde do Servidor Público Federal/ SIASS.

Com a análise de variância de Kruskal-Wallis (Figura 2) foi detectada diferença significativa no número de dias de afastamento entre os grupos $\left(c^{2}=49,9 ; \mathrm{df}=5 ; \mathrm{p}<0,001\right)$. As comparações realizadas com o teste $\mathrm{U}$ de Mann-Whitney demonstrou que o número de dias afastados no grupo "Educação" foi estatisticamente menor do que nos grupos "Segurança Pública" (U= 105,5; $\mathrm{p}<0,05)$, "Engenharia, Agricultura e Pecuária" $(U=152 ; \mathrm{p}<0,05)$ e "Recursos Florestais e 
Engenharia Ambiental" (U=31,5; $\mathrm{p}<0,05)$. O grupo "Segurança Pública" também diferiu dos grupos "Saúde e Proteção Social" ( $U=1153 ; \mathrm{p}<0,001)$ e "Jurídico Administrativa" (U=131; $\mathrm{p}<$ 0,05). O grupo "Saúde e Proteção Social” também diferiu dos grupos "Engenharia, Agricultura e Pecuária" ( $\mathrm{U}=1158 ; \mathrm{p}<0,001)$ e do grupo "Recursos Florestais e Engenharia Ambiental" (U= 338,5; p<0,001). Também foi detectada diferença significativa entre os grupos "Engenharia, Agricultura e Pecuária" e "Jurídico Administrativa" (U=195; p<0,05).

Com relação ao número de dias de afastamento para os diferentes diagnósticos, a análise de variância de Kruskal-Wallis demonstrou haver diferença estatisticamente significativa $\left(c^{2}=36,9\right.$; $\mathrm{df}=3 ; \mathrm{p}<0,01)$. As análises com o teste $\mathrm{U}$ de Mann-Whitney demonstraram que o número de dias de afastamento decorrentes de comorbidade caracterizada por diagnósticos de algum transtorno de humor e algum transtorno neurótico foi estatisticamente superior ao dos afastamentos somente por algum transtorno de humor $(U=607,5 ; \mathrm{p}<0,05)$. Não foram detectadas outras diferenças significativas.

Figura 2. Mediana do número de dias de afastamento dos grupos de acordo com os diagnósticos.

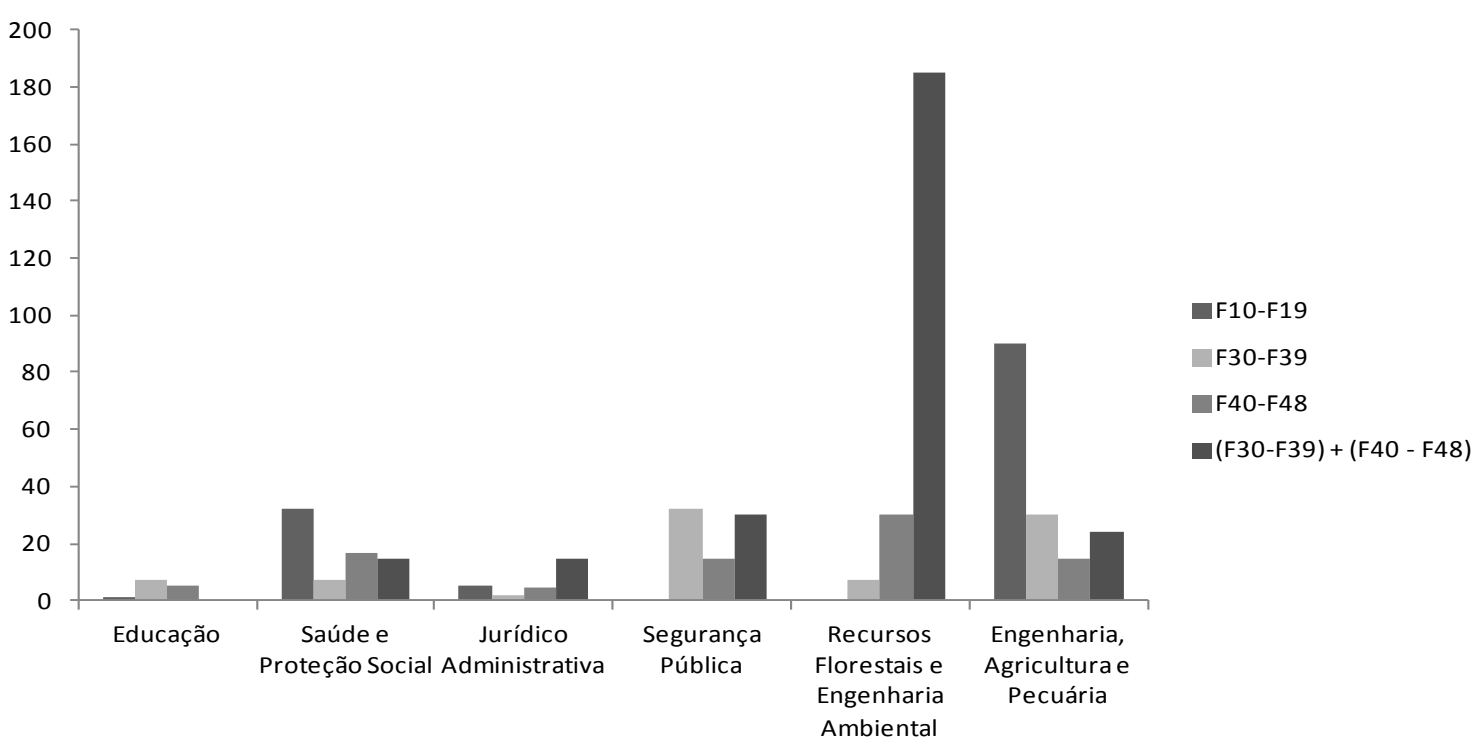

Fonte: Elaborada pelos autores, a partir dos dados obtidos no Subsistema Integrado de Atenção à Saúde do Servidor Público Federal/SIASS/RO.

\section{Discussão}

Em Rondônia, no ano de 2011, foram concedidos 3.079 afastamentos por licença saúde, aos servidores públicos federais sendo que destes, 296 (9,6\%) foram motivados por transtornos mentais e comportamentais. Os achados do estudo corroboram a prevalência de transtornos mentais e comportamentais encontrados em países industrializados, de 7\% a 30\% (Zambroni-deSouza, 2006), e em nações ocidentais variam de 7\% a 26\% (Araújo, Pinho \& Almeida, 2005), sendo superior às mulheres.

No Brasil, um estudo realizado em Alagoas sobre os afastamentos por transtornos mentais e comportamentais, em servidores públicos do Estado, 20,2\% destes foram motivados por transtornos mentais e comportamentais, em 61,0\% sobressaíram os transtornos de humor-afetivos, seguidos pelos transtornos neuróticos relacionados ao estresse e transtornos somatoformes (26,4\%) (Silva, Tomé, Costa \& Santana, 2012). Outro estudo realizado no Ceará entre os servidores públicos, no período de setembro de 2009, aponta que os afastamentos por transtornos mentais e 
comportamentais demonstraram uma alta porcentagem no período $(30,1 \%)$, sendo que a maior parte classificados no grupo F da CID-10 com diagnósticos de depressão (F32) e distúrbios ansiosos (F41). Os que mais se afastaram por esse tipo de doença foram os servidores das Secretarias do Trabalho e Desenvolvimento Social e da Educação (Nogueira, Maciel, E., Maciel, R. \& Rachel, 2010)

O estudo revelou ainda que há diferença na prevalência de afastamentos por transtornos mentais e comportamentais entre os sexos, sendo que as licenças saúde concedidas às mulheres (70,6\%) foram superiores ao número concedido aos homens (29\%), entretanto houve diferença nos diagnósticos entre os sexos. $\mathrm{O}$ uso de substâncias psicoativas foi superior entre os homens $(10,3 \%)$ em relação às mulheres $(1,4 \%)$, já para as mulheres prevaleceram os transtornos de humor associados aos transtornos neuróticos $(11,4 \%)$ em relação aos homens $(1,5 \%)$.

Sobre esse resultado, estudos epidemiológicos têm demonstrado diferenças de sexo na incidência, prevalência e curso de transtornos mentais e do comportamento. Na pesquisa realizada na Área de Captação do Hospital das Clínicas da Faculdade de Medicina da USP (Andrade, Walters, Gentil \& Laurenti, 2002), foram avaliados 1.464 indivíduos, uma amostra representativa da população geral domiciliada com idade igual ou superior a 18 anos. Neste estudo, as mulheres apresentaram maior frequência de transtornos afetivos (com exceção de episódios psicóticos de exaltação maníaca e distimia), transtornos ansiosos (exceto transtorno obsessivo-compulsivo, ansiedade generalizada e fobia social), transtornos dissociativos (transes e perdas de consciência) e transtornos alimentares. Os homens apresentaram maiores taxas de uso nocivo ou dependência de drogas, incluindo tabaco e álcool. Excluindo a dependência de tabaco, o risco de sofrer um transtorno mental durante a vida foi 1,5 vezes maior para as mulheres que para os homens.

Lennon (1995), em estudos conduzidos em comunidades, justifica que as mulheres têm apresentado consideravelmente mais sintomas de angústia psicológica e desordens depressivas que homens. Uma explicação para isto estaria na sobrecarga de trabalho da mulher com outras jornadas de trabalho como atividade doméstica e cuidados com os filhos. No estudo de Araújo, Pinho e Almeida (2005), eles descrevem a prevalência de transtornos mentais e comportamentais entre mulheres e exploram os fatores relacionados às características sociodemográficas e aspectos do trabalho doméstico, potencialmente associados aos transtornos mentais. Observaram que a mulher, mesmo após sua inserção no mercado de trabalho, permanece como a principal responsável por planejar e executar as atividades domésticas, sem receber ajuda substancial dos companheiros e filhos, sendo o auxílio nas tarefas, quando disponível, proveniente de outra mulher. Os estudos sugerem que aspectos relacionados ao trabalho doméstico podem associar as repercussões negativas à saúde mental das mulheres.

Os Transtornos do humor se caracterizam por alterações do afeto que, frequentemente, relacionam-se com situações ou fatos estressantes (OMS, 1993). Este resultado pode estar relacionado aos inúmeros fatores de estresse aos quais estão expostos estes profissionais, as mudanças sofridas no serviço público nas últimas décadas, decorrentes dos processos de modernização administrativa têm como pressuposto a descentralização do poder; adoção de critérios de qualidade para planejamento e organização do serviço público; padronização dos atendimentos; foco no cidadão usuário; simplificação de obrigações de natureza burocrática; adoção de medidas de desempenho para avaliação contínua dos serviços prestados (Prado, 2006).

No tocante ao número de licenças, o estudo demonstrou que a média de dias afastados por comorbidade (transtornos do grupo F30-F39 e do grupo F40-F48) foi de 37,4 dias (variando de 1 a 360 dias) e para os afastamentos decorrentes somente de transtornos do grupo F30-39 a média de dias afastados foi de 28,5 dias (variando entre 1 e 730 dias).

Os resultados deste estudo sinalizam uma elevada prevalência de dias de afastamento decorrentes de comorbidade caracterizada por diagnósticos de algum transtorno de humor e 
algum transtorno neurótico, sendo estatisticamente superiores aos afastamentos somente por algum transtorno de humor. De acordo com a classificação de Schilling (1984), o trabalho pode ocasionar um distúrbio latente, ou potencializar uma doença preexistente. Nesse sentido, a alta prevalência encontrada indica a necessidade de avaliar e acompanhar a incidência desse agravo em estudo com delineamento longitudinal considerando aspectos do contexto organizacional das instituições públicas do Estado.

Autores da área de Saúde Mental Relacionada ao Trabalho/SMRT compreendem que as alterações de saúde mental se dão a partir de uma gama de aspectos, desde fatores pontuais como a exposição a agentes tóxicos até a complexa articulação de fatores relativos à organização do trabalho (Jacques, 2007; Seligmann-Silva, 2011), visto que as teorias divergem sobre o papel do trabalho no processo de adoecimento mental, considerando-o ou como determinante ou como fator desencadeante, a partir de uma estrutura preexistente, mas concordam que os transtornos mentais têm uma etiologia multicausal em que conjuntos de diversos fatores interagem de modo complexo.

Cruz (2010) enfatiza a importância do estabelecimento do nexo causal, este compreende que o adoecimento resulta de um conjunto de processos que abrangem aspectos físicos e psicológicos, enfatizando a dimensão invisível destes mecanismos, que geram ao longo do tempo o agravamento das doenças ocupacionais.

\section{Considerações finais}

É importante ressaltar que, as limitações encontradas na pesquisa para a ausência de notificação dos diagnósticos, se dão em consequência da legislação vigente do Conselho Federal de Medicina - artigo 112/Resolução Conselho Federal de Medicina, no 1.658/2002, pela qual a colocação de CID, em atestado médico, só poderá ser feita mediante dever legal ou autorização expressa do paciente, sob pena do profissional violar o código de ética médica. Também, deve-se ao desconhecimento médico de determinadas doenças laborais relacionadas aos transtornos mentais e comportamentais, bem como de seu nexo causal entre o trabalho e adoecimento (Carlotto, 2010).

Salienta-se a importância do registro de todas as informações sobre as licenças saúde concedidas às instituições públicas federais de Rondônia que assinaram o termo de cooperação técnica para receber assistência à saúde no SIASS. Tais informações possibilitariam compreender melhor as relações entre o processo de adoecimento no serviço público e suas interfaces com o trabalho, com abordagem intersetorial e transdisciplinar e com a participação do principal sujeito desse processo, o servidor público. A realização de investigações mais aprofundadas, certamente, trariam mudanças na prevalência dos afastamentos, como no direcionamento e gerenciamento de políticas públicas de saúde e de prevenção, na melhoria da qualidade de vida e trabalho dos servidores públicos federais, bem como no controle de custos relacionados ao processo de reabilitação/readaptação no trabalho (Cunha, Blank \& Boing, 2009).

As informações encontradas neste estudo apontam para a necessidade urgente de planejamento, desenvolvimento e implementação da Política de Atenção à Saúde do Servidor PASS, organizada sob a forma do Subsistema de Atenção à Saúde do Servidor - SIASS, instituído pelo Decreto nº 6.833, de 29 de abril de 2009, que visualiza à manutenção da saúde do trabalhador, sua reabilitação e reintegração ao ambiente de trabalho, prevenindo, dessa forma, seu afastamento definitivo ou por longos períodos. A prevalência de transtornos mentais e comportamentais encontrada nesta investigação alerta para a magnitude do problema, evidenciando-se a urgência da implantação de ações de promoção e prevenção, para que as políticas de ação voltadas à 
melhoria das condições psicossociais do trabalho sejam mais bem direcionadas e, de fato, efetivas no setor público.

\section{Agradecimentos}

A autora agradece à Universidade Federal de Rondônia/UNIR, pelo apoio através de bolsa de PIBIC, e também às alunas, Vivian Bonfim e Cristiane Brum Marques de Mattos, que colaboraram na coleta e sistematização dos dados concedidos pelo SIASS de Rondônia.

\section{Referências}

Araújo, T. M., Pinho, P. de S. \& Almeida, M. M. G. (2005). Prevalência de transtornos mentais comuns em mulheres e sua relação com as características sociodemográficas e o trabalho doméstico. Revista Brasileira de Saúde Materno Infantil, 5(3), 337-348.

Andrade, L., Walters, E., Gentil, V. \& Laurenti, R. (2002). Prevalence of ICD-10 Mental Disorders in a Catchment Area in the City of São Paulo, Brazil. Social psychiatry and psychiatric epidemiology, 37 (7), 316-25.

Brasil. (2001). Doenças relacionadas ao trabalho: Manual de procedimentos para os serviços de saúde. Brasília: Ministério da Saúde.

Borges, L. H. (2010). Depressão. In D. M. R. Glina \& L. E. Rocha (Ed.) Saúde mental no trabalho: da teoria à prática. São Paulo: Roca.

Conselho Federal de Medicina. (2002). Lei no 1.658/2002. Recuperado em 20 de julho, 2014, de http://www.cremerrs. com.br.

Cruz, R. M. (2010). Nexo técnico e vigilância à saúde do trabalhador: uma agenda científica para o NTEP. In W. Codo (Ed.) Saúde e trabalho no Brasil: uma revolução silenciosa: o NTEP e a Previdência Social. Petrópolis: Vozes.

Cunha, J. B., Blank, V. L. G. \& Boing, A. F. (2009). Tendência temporal de afastamento do trabalho em servidores públicos (1995-2005). Revista Brasileira de Epidemiologia, 12(2), 226-236.

Carlotto, M. S. (2010). O papel do psicólogo frente ao adoecimento e sofrimento ocupacional. Pessoas e Sintomas, 11, 49-54.

Decreto n. 6.833. (2009, 29 de abril). Institui o Subsistema Integrado de Atenção à Saúde do Servidor Público FederalSIASS e o Comitê Gestor de Atenção à Saúde do Servidor. Diário Oficial [da] República Federativa do Brasil, Seção 1, p. 4.

Glina, D. M. R. (2010). Assédio moral no trabalho. In D. M. R. Glina \& L. E. Rocha (Ed.) Saúde mental no trabalho: da teoria à prática. São Paulo: Roca.

Jacques, M. da G. C. (2007). O nexo causal em saúde/doença mental no trabalho: uma demanda para a psicologia. Psicologia $\mathbb{G}$ Sociedade, 19(1), 112-119.

Lennon, M. C. (1995). Work conditions as explanations for the relation between socioeconomic status, gender, and psychological disorders. Epidemiologic Reviews, 17(1), 120-127.

McDaid, D. (2008). Mental health in workplace settings. Luxembourg: European Commission.

Ministério da Saúde. (1999). Portaria Federal nº 1.339/GM - MS, de 18 de novembro de 1999. Brasília, DF: Ministério da Saúde.

Morais, P. R. (2007). Estatística para psicólogos que não gostam de números. Santo André/SP: EseTEC.

Nogueira, C. V., Maciel, E. C., Maciel, R. H. \& Rachel de A. (2010). Afastamentos por transtornos mentais entre servidores públicos do estado do Ceará. VI Congresso Nacional em Excelência em Gestão. Ceará, CE.

Organização Internacional do Trabalho. (2010). Riesgos emergentes y nuevos modelos de prevención en un mundo de trabajo en transformación. Genebra: OIT. 
Organização Mundial da Saúde. (1993). Classificação de Transtornos Mentais e de Comportamento da Cid-10 - Descrições Clínicas e Diretrizes Diagnósticas. Porto Alegre: Artmed.

Prado, D. I. (2006). O foco é no cidadão e o atendente como fica? Contexto de atendimento presencial custo humano da atividade e qualidade de vida no trabalho. Dissertação de Mestrado em Psicologia, Instituto de Psicologia, Universidade de Brasília, Brasília.

Seligmann-Silva, E. (2009). Saúde mental no trabalho contemporâneo. In Anais do 9o Congresso Internacional de Stress da ISMA-BR. Porto Alegre/RS.

Seligmann-Silva, E. (2010). Transtornos de estresse pós-traumático. In D. M. R. Glina \& L. E. Rocha (Ed.) Saúde mental no trabalho: da teoria à prática. São Paulo: Roca.

Seligmann-Silva, E. (2011). Trabalho e desgaste mental: o direito de ser dono de si mesmo. São Paulo: Cortez.

Silva, E. B. de. F., Tomé, L. A. de. O., Costa, T. de J. G. da \& Santana, M. da C. C. P. de. (2012). Transtornos mentais e comportamentais: perfil dos afastamentos de servidores públicos estaduais em Alagoas, 2009. Epidemiologia e Serviços de Saúde, 21(3), 505-514.

Sauter, S. L., Joseph, J. H., Lawrence, R. M. \& Lennart, L. (1998). Fatores Psicosociales y de Organización. In Organização Internacional do Trabalho (OIT). Enciclopedia de Salud y Seguridad en el Trabajo. Genebra: OIT.

Schilling, R. S. F. (1984). More effective prevention in occupational health practice. Journal of the Society of Occupational Medicine, 39, 71-79.

Vieira, C. E. C. (2009). O nexo causal entre transtorno de estresse pós-traumático e trabalho: controvérsias acerca do laudo de uma perícia judicial. Revista Brasileira de Saúde Ocupacional, 34(120), 150-162.

Vieira, I. \& Jardim, S. R. (2010). Burnout reações de estresse. In D. M. R. Glina \& L. E. Rocha (Ed.) Saúde mental no trabalho: da teoria à prática. São Paulo: Roca.

Zambroni-de-Souza, P. C. (2006). Trabalhando com saúde: trabalho e transtornos mentais graves. Psicologia em Estudos, $11(1), 175-83$.

Endereço para correspondência

vdalcastel@gmail.com, moraispr@bol.com.br
Enviado em: 21/07/2013

Revisado em: 31/07/2014

Aprovado em: 11/08/2014 